\title{
Competition between Electron and Phonon Excitations in the Scattering of Nitrogen Atoms and Molecules off Tungsten and Silver Metal Surfaces
}

\author{
L. Martin-Gondre, ${ }^{1,2}$ M. Alducin,,${ }^{1,2}$ G. A. Bocan, ${ }^{3}$ R. Díez Muiño, ${ }^{1,2}$ and J. I. Juaristi ${ }^{4,1,2}$ \\ ${ }^{1}$ Centro de Física de Materiales CFM/MPC (CSIC-UPV/EHU), San Sebastián, Spain \\ ${ }^{2}$ Donostia International Physics Center DIPC, San Sebastián, Spain \\ ${ }^{3}$ Centro Atómico Bariloche, CNEA and CNICT, S. C. de Bariloche, Argentina \\ ${ }^{4}$ Departamento de Física de Materiales, Facultad de Químicas, UPV/EHU, San Sebastián, Spain
}

(Received 26 April 2011; published 28 February 2012)

\begin{abstract}
We investigate the role played by electron-hole pair and phonon excitations in the interaction of reactive gas molecules and atoms with metal surfaces. We present a theoretical framework that allows us to evaluate within a full-dimensional dynamics the combined contribution of both excitation mechanisms while the gas particle-surface interaction is described by an $a b$ initio potential energy surface. The model is applied to study energy dissipation in the scattering of $\mathrm{N}_{2}$ on $\mathrm{W}(110)$ and $\mathrm{N}$ on $\mathrm{Ag}(111)$. Our results show that phonon excitation is the dominant energy loss channel, whereas electron-hole pair excitations represent a minor contribution. We substantiate that, even when the energy dissipated is quantitatively significant, important aspects of the scattering dynamics are well captured by the adiabatic approximation.
\end{abstract}

In the last years, with the development of $a b$ initio calculations based on density functional theory (DFT), unprecedented accuracy has been achieved in describing the interaction of reactive thermal and hyperthermal gas molecules and atoms with metal surfaces. In most advanced simulations, molecular dynamics are performed in ab initio (DFT) ground state multidimensional potential energy surfaces (PES) [1]. This refined scheme relies on the validity of the adiabatic approximation. By adiabatic, we refer to a process which neglects both electronic excitations and lattice phonons excitations. Nevertheless, the role played by these nonadiabatic effects is now under close scrutiny. When the experimental results and the adiabatic results are at variance, quite often controversy arises about up to what extent the differences are due to the neglect of energy loss channels [2], to the limitations of a reduced dimensionality approximation [3], or to the inherent limitations of DFT [4-6].

The increasing number of gas-surface experiments reporting evidence of electronic excitations $[7,8]$ has motivated the development of different models to include this dissipation channel [9-12]. Among them, the local density friction coefficient approximation (LDFA) [10] offers a very good compromise between the accuracy of the results and the simplicity of its implementation [13]. Different approaches have been also developed to treat the energy exchange with the lattice [14-17]. Semiclassical approximations of the phonon excitations, in which the gassurface interaction is limited to simplified model potentials [17], have been very successful in understanding the scattering of nonreactive rare gas atoms with surfaces [18]. However, they are less accurate when applied to reactive species [19]. In the latter case, the multidimensional PES is typically so corrugated and intricate that a more realistic treatment of the interaction is required. The generalized Langevin oscillator model (GLO) [14] shows to be a sound alternative in this respect [20].

Still, the challenge in gas-surface dynamics is to provide a theoretical framework that, keeping the accuracy of a multidimensional $a b$ initio PES for the gas-metal interaction, incorporates into the dynamics energy exchange with both lattice vibrations and electronic excitations [21,22]. In this Letter we accomplish this objective by combining the GLO for phonon excitations and the LDFA for electronic excitations. The inclusion of both effects will allow us to address such fundamental questions as (i) what is the relative importance of phonon and electron-hole $(e-h)$ pair excitations as energy dissipation channels, in particular, (ii) is there any coupling between them or is their contribution just additive, and (iii) to what extent does the adiabatic calculation capture the basic physics of the dynamics and provide accurate results.

To answer these questions, we have selected two systems for which accurate energy loss measurements exist: the rotationally inelastic scattering of $\mathrm{N}_{2}$ on $\mathrm{W}(110)$ [23] and the scattering of hyperthermal $\mathrm{N}$ on $\operatorname{Ag}(111)$ [24]. Molecular and atomic nitrogen are relatively heavy projectiles for which energy exchange to the lattice can be important. The use of $\mathrm{N}$ also implies that electronic excitations are analyzed under the most favored conditions of open-shell reactive species.

The way in which we incorporate electronic and phonon excitations into the multidimensional classical trajectory simulations is as follows [25]. As in the GLO, the motion of surface atoms is represented by a three-dimensional (3D) harmonic oscillator. The latter is coupled to a 3D ghost oscillator that is subjected to frictional and random forces accounting for energy dissipation and thermal 
fluctuations [20]. The effect of electronic excitations is added by introducing for each of the impinging gas atoms a separate friction force proportional to its velocity. The electronic friction coefficient is calculated at each point of the trajectory as that of the atom moving in a homogeneous free electron gas with electronic density equal to that of the surface at this point [10]. Very recently, it has been shown that this model constitutes an efficient and sufficiently accurate tool to incorporate electronic excitations within multidimensional molecular dynamics [26].

When a rotationally cold $\mathrm{N}_{2}$ beam is scattered off $\mathrm{W}(110)$, the molecules lose around $30 \%$ of their incidence energy for low exit rotational states, but the energy loss is lower for those scattered at high exit rotational states [23]. To understand these findings, we have performed classical molecular dynamics simulations with different approximations: (i) using the adiabatic approximation, i.e., neglecting $e-h$ pair and phonon excitations, (ii) including only $e-h$ pair excitations (LDFA), (iii) including only energy exchange with the lattice (GLO) and (iv) including both energy dissipation channels (full nonadiabatic calculation with respect to both electrons and phonons). In all cases we use the 6D ab initio $\mathrm{N}_{2} / \mathrm{W}(110)$ PES of $[27,28]$, where the PW91 exchange correlation functional was used [29]. A minimum of 30000 trajectories is calculated using a conventional Monte Carlo sampling of all possible initial conditions. In (iii) and (iv), the parallel and perpendicular surface oscillator frequencies are 19 and $16 \mathrm{meV}$, respectively [30], and the friction coefficients of the ghost oscillators are obtained from the Debye frequency as proposed in [14].

In Fig. 1 we compare the results of our simulations with the experiments. The figure shows the fraction of energy retained and the energy loss as a function of the exit rotational energy for the scattered molecules, at normal incidence and detection angles. When only $e-h$ pair excitations are included, contrarily to the experimental observations, the energy loss, which is marginal, does not depend on the exit rotational energy. It only depends on the total energy no matter how it is distributed among the different degrees of freedom. The inclusion of phonon excitations changes the picture completely. We recover the experimental observation that more energy is lost at low exit rotational states. Note also that when phonon excitations are included the results with and without $e-h$ pair excitations are indistinguishable. The differences are, within the statistical errors, of the order of the contribution of $e-h$ pair excitations alone. This reflects the predominant role of phonon excitations in this kind of experiment.

The importance of including energy exchange with the lattice can be rationalized by noticing that at normal incidence and detection, though corrugation and anisotropy of the PES may complicate the picture, backscattering conditions must prevail. As a consequence, large momentum transfer takes place from the projectile to the lattice in the
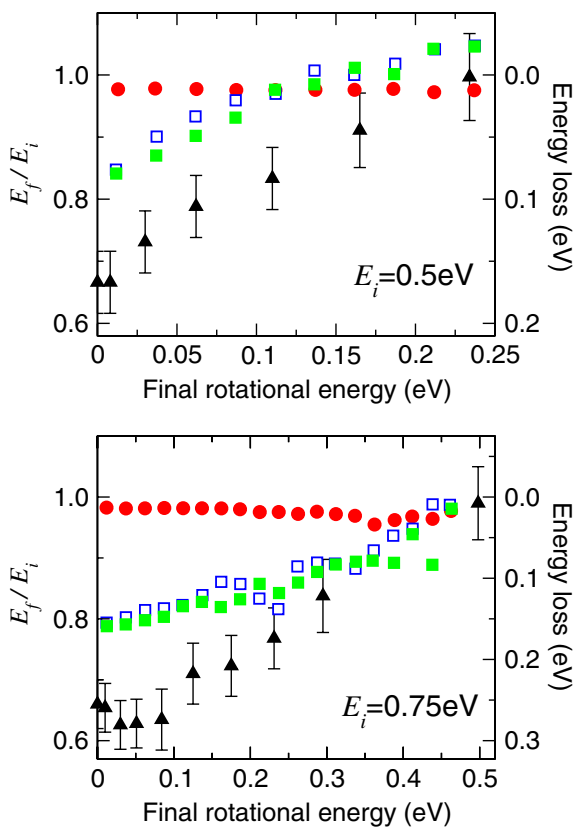

FIG. 1 (color online). Total final energy normalized to the incident energy (left ordinate) and energy loss (right ordinate) of $\mathrm{N}_{2}$ scattered off $\mathrm{W}(110)$ vs the exit rotational energy. Results for normal incidence and detection angles and two incidence energies $E_{i}$. In triangles, the experimental data of [23]. Our simulations are represented by red filled circles (LDFA), blue open squares (GLO), and green filled squares (full nonadiabatic calculation). $T_{s}=1200 \mathrm{~K}$.

direction normal to the surface. This implies a comparatively larger probability for translational energy transfer. As a result, molecules that are rotationally excited at the expense of their translational energy in the scattering with the surface, are more inefficient transferring energy to the lattice. In Ref. [15], a simplified kinematic model has been proposed to illustrate this effect. Note that at the highest rotational states, within the experimental error bars, it cannot be decided whether the molecules overcome minor energy losses or energy gains. In fact, for $E_{i}=0.5 \mathrm{eV}$ our calculations give a small energy gain consistent with the experimental error bars. In this case, the efficient conversion of translational energy into rotational energy implies a big reduction of the former, and, for the high temperatures under consideration $\left(T_{s}=1200 \mathrm{~K}\right)$, energy transfer from the lattice to the projectile is favored. The $e-h$ pair excitation mechanism cannot give rise to this kind of behavior due to the large mismatch between the projectile and the electron masses.

Interestingly, the measured rotational state population distributions of the scattered $\mathrm{N}_{2}$ are already well reproduced within the adiabatic approximation, as shown in [6]. In Fig. 2 we show that including energy exchange with lattice and $e-h$ pair excitations the width and shape of the distributions are not altered significantly. In other words, the conversion from translational to rotational energy in 


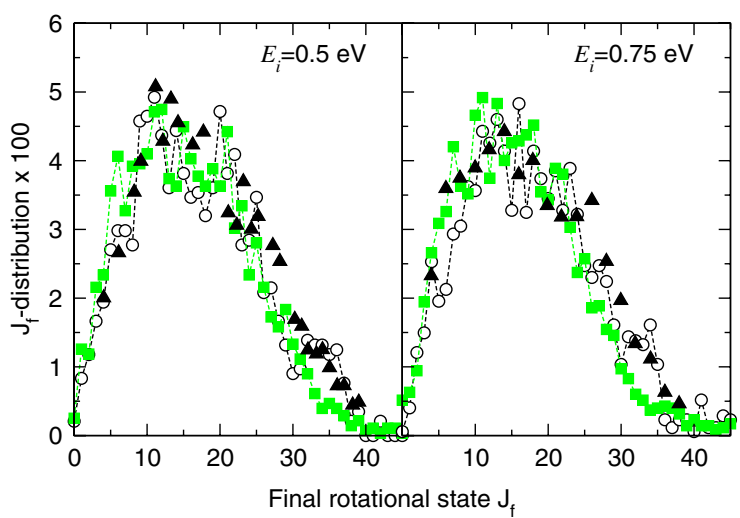

FIG. 2 (color online). Final rotational state population distributions for $\mathrm{N}_{2}$ scattered from W(110) under the same experimental conditions of Fig. 1. The experimental data of [23] (filled triangles) are compared with the adiabatic [6] (open circles) and the full nonadiabatic (green filled squares) calculations.

this kind of experiment is basically an adiabatic process, and the result of the scattering on a highly anisotropic and corrugated six-dimensional PES. Hence, even when the molecules lose a significant amount of energy, the adiabatic approximation is still valid to describe different aspects of the molecule-surface interaction, provided an accurate full-dimensional PES is used. The picture that emerges from our results is the following: in the scattering of the molecules with the anisotropic and corrugated PES, energy is transferred adiabatically from the translational to the rotational degrees of motion. Since translational energy is more efficiently transferred to the lattice than rotational energy, molecules that remain in the lowest rotational states (with higher translational energy) are the ones that lose more energy.

Next, we use our model to analyze the energy loss observed in the scattering of $\mathrm{N}$ atoms with energies of some eVs on the $\operatorname{Ag}(111)$ surface [24]. These measurements represent an excellent benchmark to explore the accuracy of our model for atoms and higher projectile energies, for which $e-h$ pair excitations are expected to be more relevant. Figure 3 reproduces the experimental results for an effusive beam of $\mathrm{N}$ scattered from $\operatorname{Ag}(111)$ at $T_{s}=500 \mathrm{~K}$ and an incidence angle $\Theta_{i}=60^{\circ}$. The beam has an average energy of $4.3 \mathrm{eV}$ and a FWHM of $\sim 5.0 \mathrm{eV}$. The figure depicts the ratio between the average final $\left\langle E_{f}\right\rangle$ and initial $\left\langle E_{i}\right\rangle$ energies as a function of the in-plane scattering angle $\Theta_{t}$ (see inset). The experiments show a decrease of the final average energy as the scattering angle increases. A remarkable feature is the dramatic increase of the energy ratio at $\Theta_{t}<60^{\circ}$, i.e., for grazing outgoing angles. In fact, the final average energy is larger than the initial one in this angular range.

We have performed classical dynamics calculations, using the $a b$ initio 3D N/Ag(111) PES of [31]. The results of our different simulations for a monoenergetic beam with

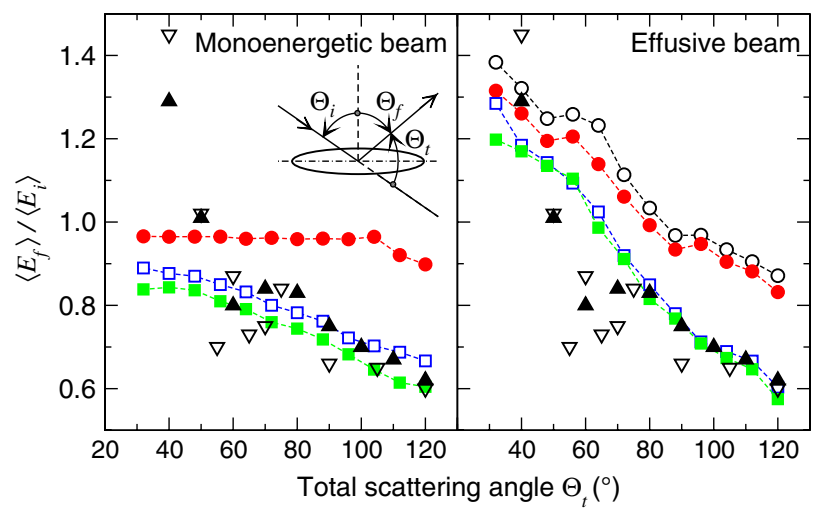

FIG. 3 (color online). Ratio of final to initial average energy vs the total scattering angle $\left[\Theta_{t}=180^{\circ}-\left(\Theta_{i}+\Theta_{f}\right)\right]$ for $\mathrm{N} / \operatorname{Ag}(111)$ at $T_{s}=500 \mathrm{~K}$ and $\Theta_{i}=60^{\circ}$. The experimental data of [24] (open and filled triangles correspond to different runs of the same experiment) are compared with our simulations for a monoenergetic (left panel) and for an effusive beam (right panel): adiabatic results (open circles), LDFA (red filled circles), GLO (blue open squares), and full nonadiabatic (green filled squares).

$E_{i}=4.3 \mathrm{eV}$ and $\Theta_{i}=60^{\circ}$ are represented in the left panel of Fig. 3. For each kind of simulation, we calculate 300000 trajectories to assure good statistics. The parallel and perpendicular surface oscillator frequencies used in the GLO and full nonadiabatic calculations are 14 and $9 \mathrm{meV}$, respectively [32]. The adiabatic results that correspond to $\left\langle E_{f}\right\rangle /\left\langle E_{i}\right\rangle=1$ are not shown in the figure. Despite the large incident energy, we observe that $e$ - $h$ pair excitations (LDFA calculations) produce marginal energy losses. In addition, the electronic energy loss is roughly independent of the scattering angle. When energy exchange with the lattice is included (GLO and full nonadiabatic calculations), the experimental results for large scattering angles are well reproduced. Again, differences between the results of these two models are of the order of the contribution of $e-h$ pair excitations alone. However, none of the energy dissipation channels is able to explain the energy ratios larger than 1 that are measured for small scattering angles. We find that this behavior is indeed due to the effusive beam itself.

mimicking the experimental effusive beam are shown in the right panel of Fig. 3. In this case, we use 600000 trajectories. For large scattering angles $\left(\Theta_{t} \geq 80^{\circ}\right)$, the results do not significantly differ from those obtained with the monoenergetic beam. However, at small scattering angles, all the effusive beam simulations give final to initial energy ratios greater than one. Quantitative agreement with the experiments is obtained only when energy exchange with the lattice is allowed, no matter whether $e-h$ pair excitations are included or not. The small discrepancy observed at $\Theta_{t} \sim 60^{\circ}$ might be due to the composition of the $\mathrm{N}$ experimental beam containing not only the ground state $\mathrm{N}\left({ }^{4} S\right)$ but also electronically excited states $\mathrm{N}\left({ }^{2} D\right)$, 
$\mathrm{N}\left({ }^{2} P\right)$, assumed to be mainly reflected around the specular position $\left(\Theta_{t}=60^{\circ}\right)[24]$.

A capture mechanism has been proposed to explain the small- $\Theta_{t}$ behavior [24]. This capture mechanism assumes that the lower energy atoms with grazing exit trajectories are trapped close to the Ag surface, resulting in the increase of the average final energy. An analysis of our simulations shows that the small- $\Theta_{t}$ behavior is due to the fact that the higher energy atoms $\left(E_{i} \geq 7 \mathrm{eV}\right)$ of the effusive beam are preferentially reflected at low scattering angles. As a result, the average energy of the atoms scattered at small (large) $\Theta_{t}$ is higher (lower) than the average energy of the incident beam. We stress that this is a pure trajectory effect that is not related to the presence or not of inelastic channels: our adiabatic simulations for the effusive beam already reproduce the correct dependence of $\left\langle E_{f}\right\rangle /\left\langle E_{i}\right\rangle$ on $\Theta_{t}$. This clearly indicates that a realistic PES is essential in order to reproduce in quantitative terms this nontrivial dynamic effect.

In summary, we have developed a theoretical framework that allows the inclusion of both phonon and $e-h$ pair excitations in a full-dimensional dynamics, keeping the accuracy of an $a b$ initio PES to treat the particle-surface interaction. Though the model is not applicable to systems in which nonadiabatic effects are due to crossing of potential energy surfaces with possible charge transfer, it can provide a proper description for a great variety of systems. Concerning the three questions posed above, our analysis shows that for two representative systems: (i) phonon excitation is the main energy dissipation channel even for the hyperthermal $\mathrm{N}$ beams, (ii) the contribution of phonon and $e-h$ pair excitations to the total energy loss is additive, and, finally, (iii) even when energy loss processes are significant, important aspects of the scattering dynamics are already captured by the adiabatic calculation: the conversion of translational to rotational energy upon scattering with the surface in the $\mathrm{N}_{2} / \mathrm{W}(110)$ case and the decrease in the number of low energy atoms that are scattered off the surface with small grazing exit angles in the $\mathrm{N} / \mathrm{Ag}(111)$ case. The correct description of these features requires a full-dimensional dynamics calculation on top of an accurate PES. In this respect, the theoretical framework proposed here is an excellent choice to efficiently include all the basic ingredients in gas-surface simulations.

This work has been supported in part by the Basque Departamento de Educación, Universidades e Investigación, the University of the Basque Country UPV/EHU (Grant No. IT-366-07) and the Spanish Ministerio de Ciencia e Innovación (Grant
No. FIS2010-19609-C02-02). Computational resources were provided by the DIPC computing center.

[1] A. Gross, in Surface Dynamics, edited by D. P. Woodruff, The Chemical Physics of Solids and Surfaces (Elsevier, Amsterdam, 2003), Vol. 11, p. 1.

[2] A. C. Luntz and M. Persson, J. Chem. Phys. 123, 074704 (2005).

[3] C. Díaz et al., Phys. Rev. Lett. 96, 096102 (2006).

[4] G. A. Bocan et al., J. Chem. Phys. 128, 154704 (2008).

[5] C. Díaz et al., Science 326, 832 (2009).

[6] K. R. Geethalakshmi et al., Phys. Chem. Chem. Phys. 13, 4357 (2011).

[7] B. Gergen et al., Science 294, 2521 (2001).

[8] J. D. White et al., Nature (London) 433, 503 (2005).

[9] J. R. Trail et al., J. Chem. Phys. 119, 4539 (2003).

[10] J. I. Juaristi et al., Phys. Rev. Lett. 100, 116102 (2008).

[11] N. Shenvi, S. Roy, and J.C. Tully, Science 326, 829 (2009).

[12] S. Monturet and P. Saalfrank, Phys. Rev. B 82, 075404 (2010).

[13] J. C. Tremblay, S. Monturet, and P. Saalfrank, Phys. Rev. B 81, 125408 (2010).

[14] J. C. Tully, J. Chem. Phys. 73, 1975 (1980).

[15] J. Kimman et al., Phys. Rev. Lett. 57, 2053 (1986).

[16] M. G. Tenner et al., Surf. Sci. 242, 376 (1991).

[17] J. R. Manson, Phys. Rev. B 43, 6924 (1991).

[18] W. W. Hayes and J. R. Manson, Phys. Rev. B 75, 113408 (2007).

[19] H. Ambaye and J. R. Manson, J. Chem. Phys. 125, 084717 (2006).

[20] H. F. Busnengo, W. Dong, and A. Salin, Phys. Rev. Lett. 93, 236103 (2004).

[21] G.-J. Kroes, Science 321, 794 (2008).

[22] E. Hasselbrink, Science 326, 809 (2009).

[23] T. F. Hanisco and A. C. Kummel, J. Vac. Sci. Technol. A 11, 1907 (1993).

[24] H. Ueta, M. A. Gleeson, and A. W. Kleyn, J. Phys. Chem. A 113, 15092 (2009).

[25] See Supplemental Material at http://link.aps.org/ supplemental/10.1103/PhysRevLett.108.096101 for a detailed description.

[26] G. Füchsel et al., Phys. Chem. Chem. Phys. 13, 8659 (2011).

[27] M. Alducin et al., Phys. Rev. Lett. 97, 056102 (2006).

[28] M. Alducin et al., J. Chem. Phys. 125, 144705 (2006).

[29] J. P. Perdew et al., Phys. Rev. B 46, 6671 (1992).

[30] M. Balden, S. Lehwald, and H. Ibach, Phys. Rev. B 53, 7479 (1996).

[31] M. S. Gravielle, G. A. Bocan, and R. Díez Muiño, Phys. Rev. A 82, 052904 (2010).

[32] M. W. G. Ponjée et al., Phys. Rev. B 67, 174301 (2003). 